\title{
Whole-transcriptome sequencing of Pinellia ternata using the Illumina platform
}

\author{
X. Huang, Y. Jing, D.J. Liu, B.Y. Yang, H. Chen and M. Li \\ Ministry of Education Key Laboratory of Standardization of Chinese Herbal \\ Medicine, College of Pharmacy, \\ Chengdu University of Traditional Chinese Medicine, Chengdu, Sichuan, China \\ Corresponding author: M. Li \\ E-mail:028limin@163.com \\ Genet. Mol. Res. 15 (2): gmr.15028062 \\ Received November 17, 2015 \\ Accepted February 12, 2015 \\ Published July 15, 2016 \\ DOI http://dx.doi.org/10.4238/gmr.15028062
}

\begin{abstract}
Pinelliae rhizoma is the dried tuber of Pinellia ternata (Thunb.) Breit., and has been used for thousands of years as a traditional Chinese medicine. However, its genomic background is little known. With the development of high-throughput genomic sequencing, it is now easy and cheap to obtain genomic information. In this study, 193,032,910 high-quality clean reads were generated using the Illumina Hiseq 2000 platform. A total of 53,544 unigenes were identified from the contigs assembled. Functional annotation analysis annotated $37,318,27,697,23,043,22,869,23,328$, and 27,415 unigenes. KEGG analysis revealed that five pathways (169 genes) were associated with alkaloid synthesis, 201 unigenes were related to fatty acid biosynthesis (ko00061), and 133 unigenes were involved in the biosynthesis of unsaturated fatty acids (ko01040). In addition, 6703 simple sequence repeats were designed based on the unigene sequences for screening germplasm resources in the future. These data are a valuable resource for genomic studies on Pinellia plants.
\end{abstract}

Key words: Pinellia ternata; Next-generation sequencing; Organic acids; Alkaloid 


\section{INTRODUCTION}

Pinelliae rhizoma is the dried tuber of Pinellia ternata (Thunb.) Breit. (Figure 1). It has been widely used in Chinese medicine for drying dampness and resolving phlegm, suppressing Qi to stop vomiting, relieving stuffiness, dissipating nodulations, and relieving swelling (Chinese Pharmacopoeia Commission, 2015) by influencing the respiratory, digestive, cardiovascular, and nervous systems. It is anticarcinogenic, anti-inflammatory, and antibacterial (Li et al., 2004). P. ternata is widely distributed in China, except in Neimenggu, Jilin, Heilongjiang, Xinjiang, Qinghai, and Tibet Provinces (Li et al., 2009). It is mainly cultivated in Gansu, Shanxi, Shandong, Guizhou, and Yunnan Provinces (Huadong and Faming, 2012). Because of its wide distribution in China, it is difficult to evaluate its quality.

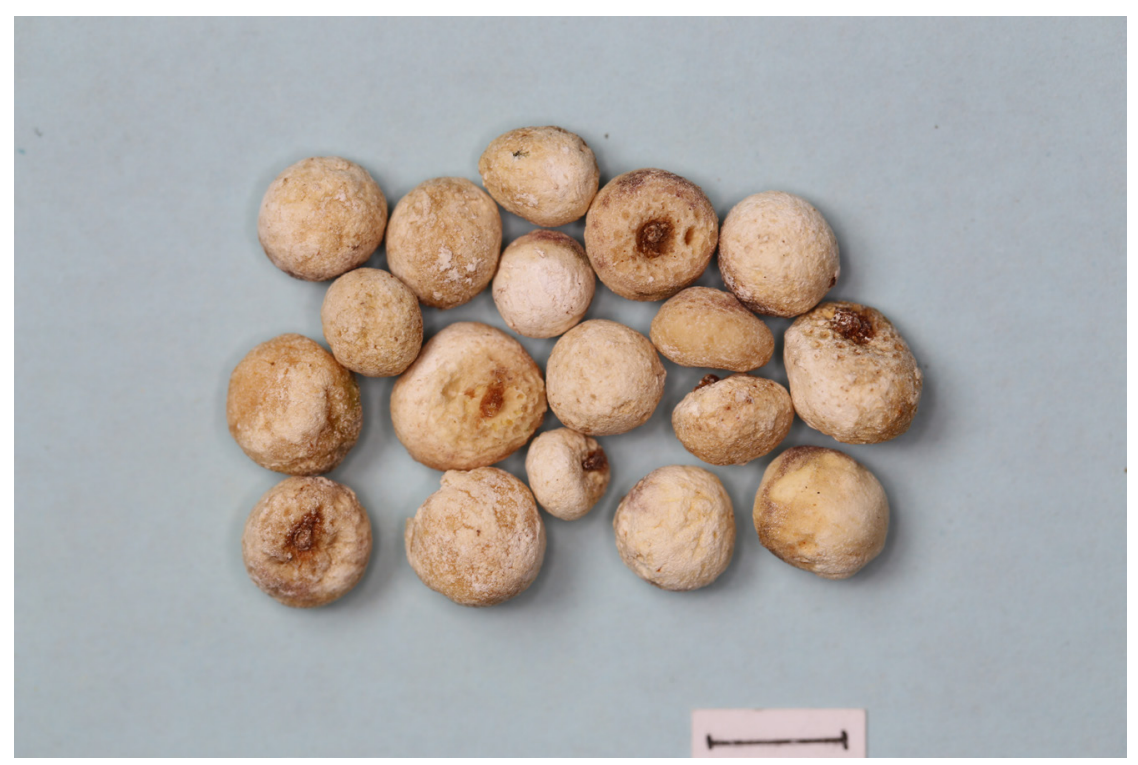

Figure 1. Pinellia medicinal materials.

Pinelliae rhizoma contains alkaloids, sterols, organic acids, amino acids, inorganic elements, and nucleosides (Han et al., 2006). Organic acids are regarded as important for evaluating the quality of $P$. ternata (Chinese Pharmacopoeia Commission, 2015), and alkaloids exhibit significant biological and pharmacological activity. Many factors affect the quality of $P$. ternata, including the place of origin, timing of the harvest, germplasm, light, and processing methods.

In recent years, researchers have focused on identifying the genetic diversity of $P$. ternata. The genetic control of heterosis for guanosine and organic acid content has been reported (Luo et al., 2013), and 12 microsatellite markers have been identified that could provide a useful tool for studying the genetic structure of Pinellia species (Chen et al., 2008). Inter-simple sequence repeat (ISSR) and sequence-related amplified polymorphism (SRAP) markers have been used to analyze genetic relationships among the Pinellia in China (Liu et al., 2012), and random amplified polymorphic DNA (RAPD) and polymerase chain reactionrestriction fragment length polymorphism (PCR-RFLP) have been employed to authenticate 
Chinese and Korean herbal medicines (Chung et al., 2002). A total of 54 microRNAs (miRNAs) belonging to 23 miRNA families have been identified (Xu et al., 2012); however, no studies have investigated associations between genes and phenotypic quality in P. ternata, or its DNA and protein sequences. Indeed, little is known about what the genes encode in the species. As of October 2015, only 387 nucleotide sequences, 353 proteins, and 131 genes from P. ternata have been deposited in the GenBank database of National Center for Biotechnology Information (NCBI). Transcriptome analysis is a useful and cost-effective method for discovering new genes and providing information on gene expression and regulation (Margulies et al., 2005; Huse et al., 2007; Novaes et al., 2008). In this study, we surveyed the poly (A) + transcriptome of $P$. ternata using next-generation sequencing technology. We obtained $17.33 \mathrm{~Gb}$ of raw reads, and Kyoto Encyclopedia of Genes and Genomes (KEGG) annotation revealed five pathways (169 genes) that were associated with alkaloid synthesis. A total of 201 unigenes were related to fatty acid biosynthesis (ko00061) and 133 unigenes were involved in the biosynthesis of unsaturated fatty acids (ko01040). These data can be used to study alkaloid, saturated fatty acid, and unsaturated fatty acid synthesis at the gene level in P. ternata, and reveal associations between genes and chemical components. They will also provide research ideas on virus infection, the death of improved varieties, the low coefficient of natural reproduction, the cultivation of low technology, and counterfeit $P$. ternata plants.

\section{MATERIAL AND METHODS}

\section{Plant materials and RNA isolation}

P. ternata plants were planted in traditional Chinese medicine-producing areas of the town of Hongjiang (Peng xi County, Suining City, Sichuan Province, China; $30.69^{\circ} \mathrm{N}, 105.45^{\circ} \mathrm{E}$ ) (Figure 2). We selected three strains as one biological replication with three replications (each sample is about $0.5 \mathrm{~g}$ ) in September 2014, when the plants were growing. After selection, the specimens were washed with pure water, dried using filter paper, and small pieces were cut on ice and immediately frozen in liquid nitrogen. All of the samples were stored at $-80^{\circ} \mathrm{C}$ until analysis.

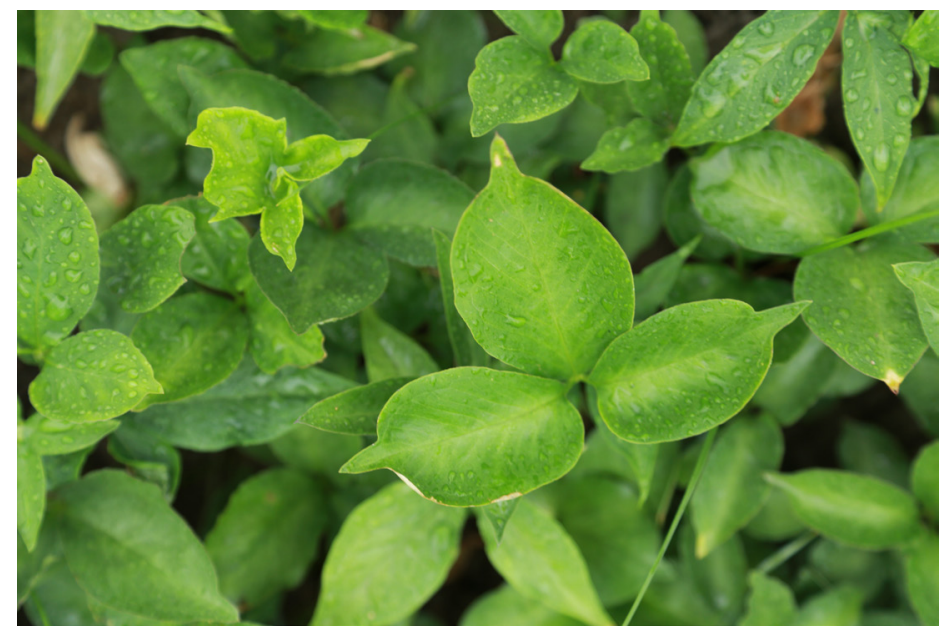

Figure 2. Pinellia ternata plant. 
Total RNA was isolated from each sample using TRIzol (Invitrogen) following the manufacturer instructions. RNase-free DNase I (New England BioLabs) for $30 \mathrm{~min}$ at $37^{\circ} \mathrm{C}$ was used to expurgate residual DNA from the total RNA. Equal amounts of RNA from each sample were prepared for mRNA-seq library construction.

\section{RNA-seq library construction for Hiseq 2000 sequencing}

Total RNA was purified using Oligo(dT) beads (Dynabeads ${ }^{\circledR}$ mRNA Purification Kit, Invitrogen), then poly (A)-containing mRNA was fragmented into 200-250 bp with Fragment Buffer (Ambion). First-strand cDNA was generated using an N6 primer, First-Strand Master Mix, and Super Script II Reverse Transcriptase (Invitrogen). The reaction conditions included $25^{\circ} \mathrm{C}$ for $10 \mathrm{~min}, 42^{\circ} \mathrm{C}$ for $30 \mathrm{~min}$, and $70^{\circ} \mathrm{C}$ for $15 \mathrm{~min}$, with a $4^{\circ} \mathrm{C}$ hold. Second-Strand Master Mix was then added to synthesize the second-strand cDNA $\left(16^{\circ} \mathrm{C}\right.$ for $\left.2 \mathrm{~h}\right)$, the cDNA was purified using a QIAquick ${ }^{\circledR}$ PCR Purification Kit (Qiagen), End Repair Mix was added, and it was incubated at $20^{\circ} \mathrm{C}$ for $30 \mathrm{~min}$. It was then purified using A-Tailing Mix and incubated at $37^{\circ} \mathrm{C}$ for $30 \mathrm{~min}$. The purified Adenylate 3'-Ends DNA, Adapter and Ligation Mix was combined, and the ligation reaction was incubated at $20^{\circ} \mathrm{C}$ for $20 \mathrm{~min}$. A $2 \%$ agarose gel was then run in order to select a narrow 300-350-bp size range, and the gel was purified using a QIAquick ${ }^{\circledR}$ Gel Extraction Kit (Qiagen). Several rounds of PCR amplification using PCR Primer Cocktail and PCR Master Mix were performed to enrich the cDNA fragments. The PCR products were then purified with AMPure XP Beads (Agencourt). The final library was quantitated in two ways: average molecule length was determined using an Agilent 2100 Bioanalyzer (Agilent DNA 1000 Reagents), and the library was quantified by PCR (TaqMan ${ }^{\circledR}$ Probe). The quantified libraries were amplified on cBot to generate a cluster on a flow cell (TruSeq PE Cluster Kit v3-cBot-HS, Illumina). Finally, the amplified flow cell was paired-end sequenced on the HiSeq 2000 System (TruSeq SBS KIT-HS V3, Illumina). A read length of 90 was the most common sequencing strategy.

\section{Sequence data assessment and assembly}

Clean reads were obtained after removing adaptors and low-quality reads (the proportion of reads with a quality value of 10 or less was over $20 \%$ ). The CycleQ20 value was used to evaluate the clean reads. The de novo transcriptome assembly of the short clean reads was conducted using Trinity (release-20130225, http://trinityrnaseq.sourceforge.net/), which recovers full-length transcripts across a broad range of expression levels with a sensitivity similar to methods that rely on genome alignments (Grabherr et al., 2011). The overlap settings used for the assembly were $25 \mathrm{bp}$ and $70 \%$ similarity.

\section{Unigene annotation}

The assembled unigenes were compared against the NCBI non-redundant protein (Nr) and Swiss-Prot databases using BLASTx (version 2.2.14), with an E-value lower than $10^{-5}$. Gene names were assigned to each assembled sequence based on the best BLAST hit (highest score). Blast2GO (Conesa et al., 2005) was then used to obtain a Gene Ontology (GO) annotation of the unigenes. For each unigene within a GO annotation, the WEGO software (Ye et al., 2006) was used to obtain the GO functional classification for the unigenes, and to 
ascertain the distribution of gene functions of the species at the macro-level.

Unigenes were aligned to the Clusters of Orthologous Groups (COGs) database to predict and classify possible functions (Tatusov et al., 2000). KEGG pathways were assigned to the assembled unigenes using the online KEGG Automatic Annotation Server (http://www. genome.jp/kegg/kaas/).

\section{Expressed sequence tag-simple sequence repeat (EST-SSR) detection}

By using the unigenes as references, SSRs were detected using the MicroSAtellite (MISA) program (Temnykh et al., 2001). Perfect di-, tri-, tetra-, penta-, and hexanucleotide motifs were adjusted to identify the parameters with a minimum of six, five, five, four, and four repeats, respectively. Among the submitted unigenes, we obtained a number of sequences containing SSRs, sequence IDs, SSR motifs, repeats (di-, tri-, tetra-, penta-, and hexanucleotide repeat units), repeat lengths, SSR starts, and SSR ends (Yu et al., 2004; Varshney et al., 2005a,b).

\section{RESULTS}

\section{P. ternata transcriptome sequencing and de novo assembly}

Three parallel samples of $P$. ternata were collected and sequenced on the Illumina Hiseq 2000. After removing the adaptors and low-quality reads (Q20, 20), 193,032,910 highquality clean reads that were $75 \mathrm{bp}$ long were obtained (Table 1). All of the high-quality reads were assembled de novo using Trinity (Grabherr et al., 2011), which produced 189,150, 72,615 , and 52,419 contigs from P1, P2, and P3, respectively, with an N50 of 214, 472, and 452 bp, respectively.

Table 1. Summary of the sequencing and de novo assembly of the Pinellia ternata transcriptome.

\begin{tabular}{l|c|c}
\hline Transcript length & Total number & Percentage \\
\hline $100-500$ & 33,929 & 23.37 \\
\hline $500-1000$ & 10,744 & 8.07 \\
\hline $1000-1500$ & 4616 & 4.11 \\
\hline $1500-2000$ & 2201 & 3.84 \\
\hline$\geq 2000$ & 2054 & \\
\hline Total number & 53,544 & \\
\hline Total length & $32,716,910$ & \\
\hline N50 length & 907 & \\
\hline Mean length & 611 & \\
\hline
\end{tabular}

The size distributions of the contigs are presented in Figure 3A-C. The contigs were then assembled into unigenes, the size distributions of which are presented in Figure 3D-F. Although the unigene distributions closely matched the contig distributions with the majority being short sequences, we obtained more long unigenes than long contigs (Figure 3A-C). We obtained 53,544 unigenes with an average length of 611 bp and an N50 of $907 \mathrm{bp}$ (Figure 3G). Of these, 19,615 (36.63\%) were longer than $500 \mathrm{bp}, 8871$ (16.57\%) were longer than 1000 $\mathrm{bp}$, and 4255 (7.95\%) were longer than $1500 \mathrm{bp}$. Although the majority of the unigenes were smaller than $500 \mathrm{bp}, 2054$ (3.84\%) were greater than $2000 \mathrm{bp}$ in length. The longest unigene was CL823.Contig2_All that was 18,172 bp long, and according to the COG annotation encodes outer membrane proteins during cell wall/membrane/envelope biogenesis. 

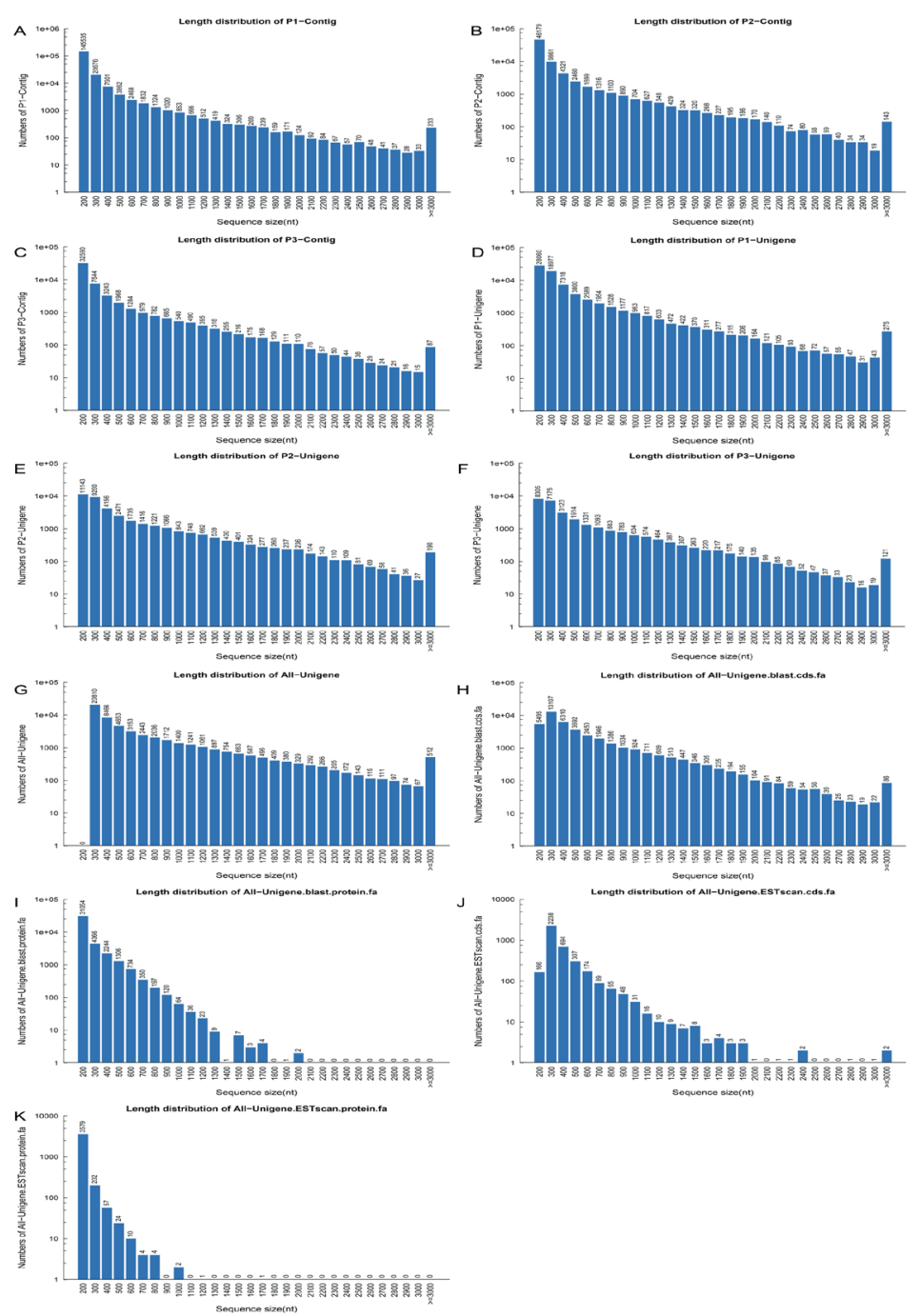

Figure 3. Overview of Pinellia ternata transcriptome assembly. A.-C. Size distributions of the contigs; D.-G. size distributions of the unigenes; H. size distributions of the coding DNA sequences; I. size distributions of the proteins; J. and K. size distributions of the expressed sequence tags and proteins.

In order to analyze the coding DNA sequences (CDSs) and gene annotations, all of the assembled unigenes were first aligned in BLASTx (E-value, $10^{-5}$ ) to protein databases in the order Nr, Swiss-Prot, KEGG, and COG, i.e., we first aligned the unigenes to Nr, then SwissProt, then KEGG, and finally to COG (Natale et al., 2000). We obtained 42,687 significant BLAST hits (79.72\% of all unigenes). The size distributions of the CDSs and predicted proteins are presented in Figure $3 \mathrm{H}$ and I. Assembled unigenes that could not be annotated by any database were predicted by ESTScan, and were then translated into peptide sequences (Iseli et al., 1999); 3884 unigenes were analyzed by ESTScan. The size distributions of the ESTs and proteins are presented in Figure 3J and K. 


\section{Functional annotation}

\section{Nr annotation}

Almost $70.70 \%(37,318)$ of the unigenes were annotated using the $\mathrm{Nr}$ database. The highest homology (E-value $<10^{-100}$ ) of the unigenes was $16.1 \%$ and the moderate homology $\left(10^{-100}<\right.$ E-value $\left.<10^{-60}\right)$ was $14.1 \%$ (Figure $4 \mathrm{~A}$ ). A total of $38.1 \%$ of the sequences had a higher similarity than $80 \%, 27.6 \%$ had moderate similarities of approximately $60-80 \%$, and $22.8 \%$ had a similarity between $40-60 \%$ (Figure $4 \mathrm{~B}$ ). For the species distribution of aligned hits, $18.6 \%$ of the hits had top matches to sequences from Japanese rice, $15.3 \%$ to sequences from Vitis vinifera, and 8.2\% to sequences from Cucumis sativus (Figure 4C). Therefore, the assembled transcripts were high quality.

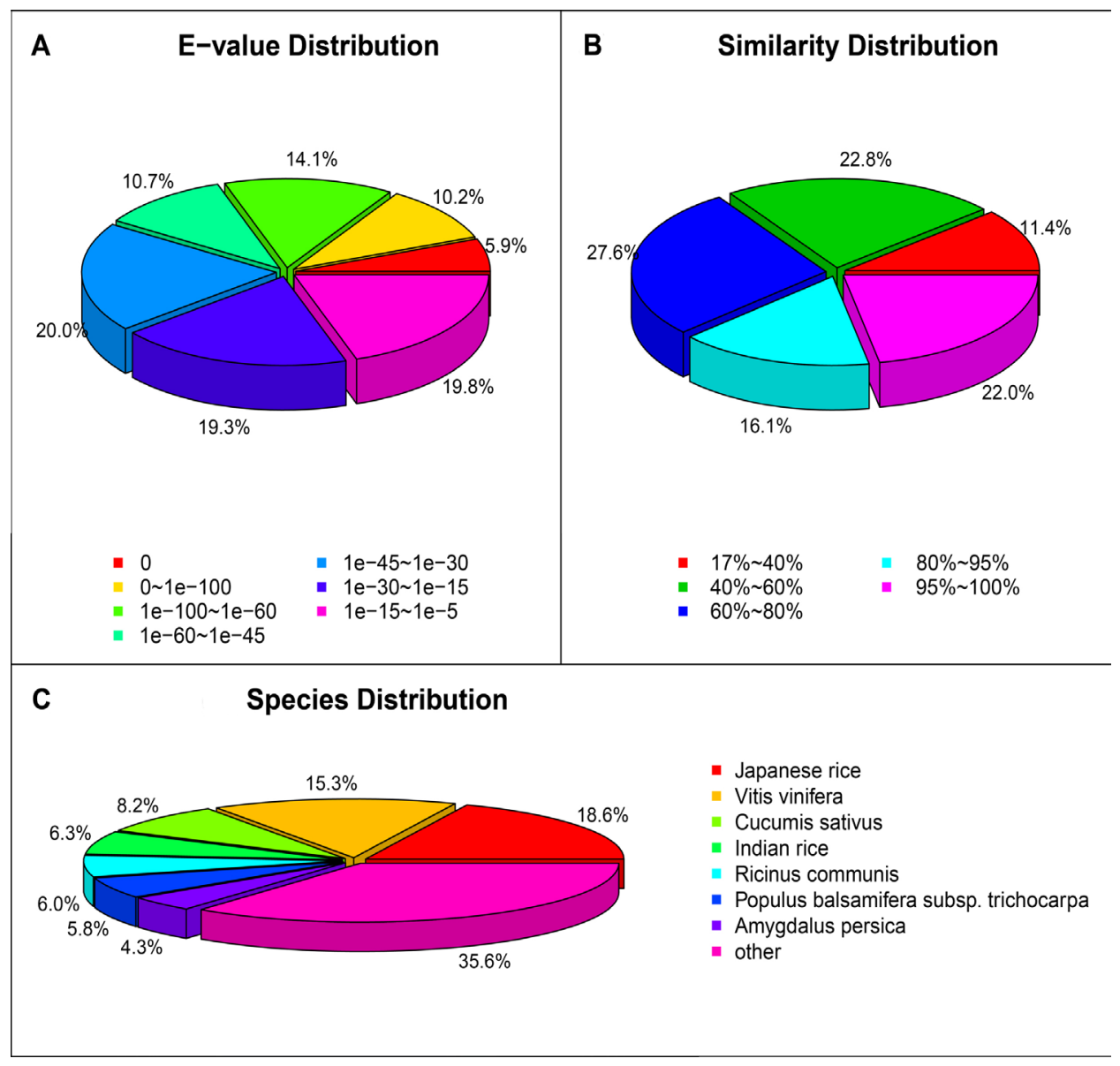

Figure 4. Characteristics of a homology search of Pinellia ternata unigenes. A. E-value distribution of the top BLASTx hits against the non-redundant $(\mathrm{Nr})$ protein database for each unigene; $\mathbf{B}$. similarity distribution of unigene BLASTx hits against the $\mathrm{Nr}$ database; $\mathbf{C}$. number of unigenes matching the top seven species using BLASTx in the $\mathrm{Nr}$ database. 


\section{COG classification}

A total of 23,328 unigenes were annotated to 25 functional categories using COG (Figure 5A). "General function prediction only" was the largest COG category (22.81\%), followed by "translation, ribosomal structure, and biogenesis" (17.15\%), "transcription" (16.92\%), "replication, recombination, and repair" (11.71\%), "posttranslational modification, protein turnover, chaperones" $(11.15 \%)$, "signal transduction mechanisms" (11.01\%), "amino acid transport and metabolism" (10.76\%), "carbohydrate transport and metabolism" (10.70\%), "lipid transport and metabolism" (5.04\%), and "secondary metabolites biosynthesis, transport, and catabolism" (4.69\%). The smallest groups were "nuclear structures" (10 unigenes) and "extracellular structures" (nine unigenes) (Figure 5A).

\section{A}

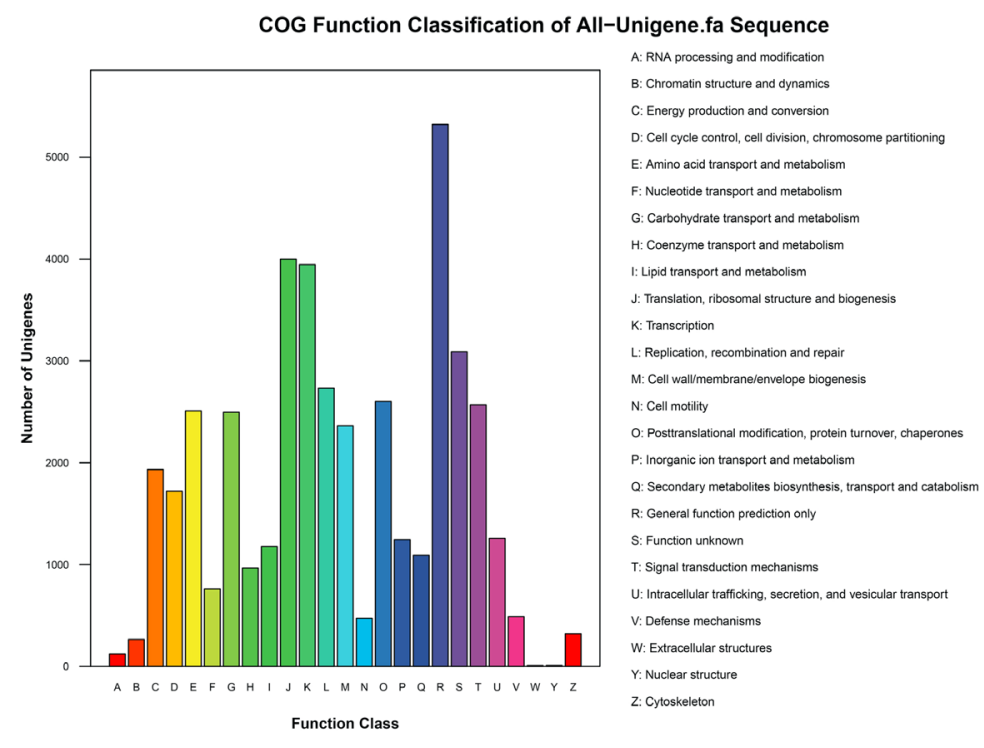

B

All-Unigene GO Classification

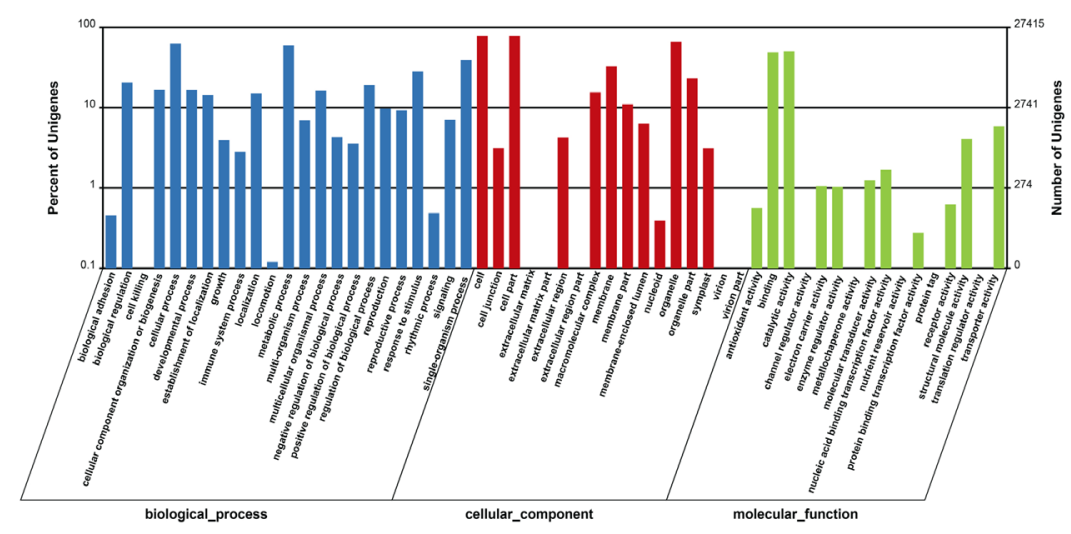

Figure 5. Clusters of Orthologous Groups (COGs) functional classification and Gene Ontology (GO) classification of the Pinellia ternata transcriptome. 


\section{GO classification}

A total of 27,415 unigenes were assigned 5043 GO-term annotations using BLAST2GO, and the terms were summarized into the three main GO categories and 56 subcategories (functional groups) (Figure 5B). In the "biological processes" category, a high percentage of genes were involved in "cellular processes" (62.30\%) and "metabolic processes" (59.74\%), followed by "single-organism processes" (39.09\%) and "response to stimulus" (28.18\%). For the "cellular components" category, the dominant terms were "cells" (77.93\%), "cell parts" (77.92\%), and "organelles" (65.87\%); "virion" and "virion parts" contained only 17 genes. In the "molecular function" category, the main terms were "binding" (48.87\%) and "catalytic activity" $(50.30 \%)$, followed by "transporter activity" (5.84\%) and "structural molecule activity" (4.06\%) (Figure 5B).

\section{KEGG pathway mapping}

We found 128 KEGG pathways with 22,869 unigenes (the main pathways were "metabolic pathways" (6577, 28.76\%) and "biosynthesis of secondary metabolites" (2872, $12.56 \%$ ). Moderate numbers of unigenes were mapped to the following pathways: "RNA transport" (1707, 7.46\%), "mRNA surveillance" (1253, 5.48\%), "endocytosis" (1155, 5.05\%), "ribosome" (1090, 4.77\%), "glycerophospholipid metabolism" (1060, 4.64\%), "spliceosome" (1038, 4.54\%), "ether lipid metabolism" (932, 4.08\%), "plant hormone signal transduction" $(864,3.78 \%)$, and "plant-pathogen interactions" $(854,3.73 \%)$. Only five unigenes were assigned to "betalain biosynthesis", and one unigene was assigned to "D-Arginine and D-ornithine metabolism".

\section{SSR discovery}

The MISA analysis identified 6703 putative microsatellites from 5525 unigenes $(10.32 \%)$. Of these, seven types of SSR were discovered and 926 sequences contained more than one SSR. The most abundant was the trinucleotide repeat motif, which accounted for $39.82 \%$, followed by dinucleotide (30.87\%), mononucleotide $(26.21 \%)$, tetranucleotide $(1.22 \%)$, pentanucleotide $(0.78 \%)$, and hexanucleotide repeats $(1.10 \%)$ (Table 2).

Table 2. Summary of simple sequence repeat (SSR) types in Pinellia ternata.

\begin{tabular}{l|c}
\hline Search item & Number \\
\hline Total number of sequences examined & 53,544 \\
\hline Total number of SSRs identified & 6703 \\
\hline Number of SSRs containing sequences & 5525 \\
\hline Number of sequences containing more than one SSR & 926 \\
\hline Number of SSRs present in compound formation & 520 \\
\hline Mononucleotide & 1757 \\
\hline Dinucleotide repeats & 2069 \\
\hline Trinucleotide & 2669 \\
\hline Tetranucleotide & 82 \\
\hline Pentanucleotide & 52 \\
\hline Hexanucleotide & 74 \\
\hline
\end{tabular}




\section{DISCUSSION}

Carles et al. (2005) used a silicon-based DNA microarray to identify toxic Chinese medicines associated with $P$. ternata, and found that many have a similar genetic heritage. By directly sequencing $P$. ternata and its adulterant $P$. pedatisecta, Schott's nuclear genome sequence provides a molecular basis for the original identification of $P$. ternata. Lin et al. (2006) used special DNA primers by PCR to identify different types of $P$. ternata and its adulterants, and found that two specific sites could be identified by the restriction endonucleases Bam HI and NcoI in the opening DNA sequence. RAPD and PCR-RFLP have also been used to authenticate Chinese and Korean herbal medicines (Chung et al., 2002). In the present study, we obtained a large amount of genetic information using transcription, and a great deal of SSR information (6703 SSRs). It is now possible to develop cDNA SSR (cSSR) tags for breeding by transcriptome sequencing (Wang et al., 2010), and EST-SSR markers from transcriptome sequencing data (Li et al., 2012; Luo et al., 2015). Using the $P$. ternata transcriptome sequencing data, cSSRs can be developed that can be used in genetic mapping, molecular marker-assisted selection, variety identification, etc. Studies of $P$. ternata genes and their components have mainly focused on Pinellia proteins, which are involved in clotting, fertility, tumor resistance, and insect resistance. Yao et al. (2003b) first reported the $P$. ternata lectin gene and coded the amino acid sequence. They imported Pinellia protein genes into tobacco, which were successfully expressed in response to attack by homopteran pests such as aphids (Yao et al., 2003a). SJ-10 was cloned from the P. ternata agglutinin (PTA) gene and inoculated into rice plants, which then exhibited insecticidal activity against the white-backed planthopper, Sogota furcifera Horvath (Zhang et al., 2011). In our study, 3884 assembled unigenes that could not be aligned to any database were predicted by ESTScan and then translated into peptide sequences. Using KEGG, 2417 unigenes were mapped to the "amino acid metabolism" pathway and 536 to the "metabolism of other amino acids" pathway. There were 13 pathways in the "amino acid metabolism" pathway, and most unigenes were in the "glycine, serine, and threonine metabolism" pathway (293 unigenes, $12.12 \%$ ) followed by the "cysteine and methionine metabolism" pathway (278 unigenes, $11.50 \%$ ); only 73 unigenes were aligned to the "lysine biosynthesis" pathway. Regarding the "metabolism of other amino acids" pathway, 186 unigenes $(34.70 \%)$ were mapped to the "glutathione metabolism" pathway and only one unigene was mapped to the "D-Arginine and D-ornithine metabolism" pathway. These data will provide a reference for research on Pinellia proteins and their relationships with genes.

P. ternata genes involved in alkaloids and organic acids have been little studied. Liu et al. (2010a,b) investigated the potential use of protocorm-like bodies (PLBs) for studying alkaloid metabolism in P. ternata by investigating the induction and in vitro alkaloid yield of calluses and PLBs. Organic acids are one of the active ingredients of Pinelliae rhizoma, which contains a large amount of unsaturated fatty acids (49.15\%) that include linoleic acid (37.10\%); the saturated fatty acids present include palmitic acid (15.16\%) (Kewei et al., 2002). In the KEGG analysis, 201 unigenes were related to fatty acid biosynthesis (ko00061) and 133 were involved in the biosynthesis of unsaturated fatty acids (ko01040). These genes will provide a reference for research into total organic acid biosynthesis in $P$. ternata.

Only 1093 unigenes $(4.69 \%)$ were related to secondary metabolite biosynthesis, transport, and catabolism in the COG classification. Twelve pathways were found in the KEGG classification ( $9.38 \%$ of the total number of pathways). In these pathways, five unigenes were 
involved in betalain biosynthesis (ko00965) and nine were related to caffeine metabolism (ko00232). Eleven unigenes were involved in indole alkaloid biosynthesis (ko00901), 66 were involved in isoquinoline alkaloid biosynthesis (ko00950), and 78 were involved in tropane, piperidine, and pyridine alkaloid biosynthesis (ko00960). All of these alkaloid metabolismrelated genes are potential candidate genes for alkaloid synthesis in P. ternata.

There are few data available regarding $P$. ternata genome sequences. Researchers have focused on the complete chloroplast genome sequence of $P$. ternata, which has the potential to advance population and phylogenetic studies of this plant (Han et al., 2015). Suppression subtractive hybridization has been used to clone transcripts that are upregulated during heat stress in P. ternata leaves (Lu et al., 2013). Fifteen cDNA fragments have been obtained from the small tubers of $P$. ternata, which probably play an important role during tuber development (Yueqin, 2008).

De novo transcriptome sequencing is the transcriptome profiling of organisms or species based on high-throughput technologies without the aid of a reference genome, and includes library construction, sequencing, and bioinformatic analysis. To our knowledge, this is the first de novo sequencing of three parallel samples from $P$. ternata using an Illumina Hiseq 2000. The sequencing produced 193,032,910 clean reads and 53,544 unigenes after assembly. The number of unigenes obtained from P. ternata was lower than that obtained from Carthamus tinctorius (120,778) (Lulin et al., 2012), Polygonum cuspidatum $(86,418)$ (Hao et al., 2012), and Salvia miltiorrhiza $(64,139)$ (Yang et al., 2013), However, the number of unigenes obtained from P. ternata was higher than that from Codonopsis pilosula $(45,511)$ (Gao et al., 2015), and Isatis indigotica (36,367) (Chen et al., 2013). The average unigene length (611 bp) was slightly greater than that of other medicinal plants, including C. tinctorius (446 bp) (Lulin et al., 2012), S. miltiorrhiza (413 bp) (Yang et al., 2013), and P. cuspidatum (365 bp) (Hao et al., 2012). The unigene annotation rate observed in the present study (79.72\%) was relatively higher. It is noteworthy that 8871 unigenes were longer than $1000 \mathrm{bp}$ and accounted for $16.57 \%$ of all the unigenes, indicating that the Illumina Hiseq 2000 can provide a highquality transcriptome library and reliable global screening of functional genes in non-model plants, particularly medicinal plants.

According to the $\mathrm{GO}$ classification, the unigenes were related to biological processes (75.62\%), cellular components (81.03\%), and molecular functions (76.19\%). A total of 16,378 unigenes were related to metabolic processes, including secondary metabolite biosynthesis, transport, and catabolism. The active ingredients in P. ternata may be related to secondary metabolite biosynthesis. There were also high numbers of unigenes that were linked to "response to stimulus" and "signaling", demonstrating that $P$. ternata can rapidly adapt to changes in the natural environment.

In this study, we obtained 6703 putative microsatellites that can be used to investigate the population-level diversity of molecular markers in future studies. For designing primers and developing nuclear gene markers, unigenes related to low-copy nuclear genes can be used, which is important for studying plant phylogenies. The functions of $3089(5.77 \%)$ unigenes were unknown; therefore, future studies are needed to elucidate these unigenes. To our knowledge, this is the first time that the Illumina Hiseq 2000 platform has been used for three parallel samples of $P$. ternata. It produced about 53,544 unigenes, $69.70 \%$ of which were aligned to the $\mathrm{Nr}$ database. A total of 1093 unigenes (4.69\%) were aligned to secondary metabolite biosynthesis, transport, and catabolism (12 pathways), and 6703 SSRs were identified that will promote the development of the marker. These data provide insights into 
the genetic diversity of $P$. ternata, and provide a better understanding of alkaloid and organic acid biosynthesis in $P$. ternata at the genetic level. In conclusion, these data will provide a new resource for genomic studies on the main chemical components of $P$. ternata.

\section{Conflicts of interest}

The authors declare no conflict of interest.

\section{ACKNOWLEDGMENTS}

The authors would like to thank the Beijing Genomics Institute (Shenzhen) for assistance with raw data processing and the related bioinformatic analysis. We thank the anonymous referees and editors for carefully reviewing our manuscript and providing constructive comments. Research supported by the National Natural Science Fund Project (\#81173492) and the Department of Sichuan Province "Twelfth Five Year" Crop Breeding Research (\#2011NZ0098-12).

\section{REFERENCES}

Carles M, Cheung MK, Moganti S, Dong TT, et al. (2005). A DNA microarray for the authentication of toxic traditional Chinese medicinal plants. Planta Med. 71: 580-584. http://dx.doi.org/10.1055/s-2005-864166

Chen F, Zhang L and Zhao C (2008). Isolation and characterization of microsatellite markers for Pinellia ternata and cross-species amplification. Mol. Ecol. Resour. 8: 1460-1462. http://dx.doi.org/10.1111/j.1755-0998.2008.02326.x

Chen J, Dong X, Li Q, Zhou X, et al. (2013). Biosynthesis of the active compounds of Isatis indigotica based on transcriptome sequencing and metabolites profiling. BMC Genomics 14: 857. http://dx.doi.org/10.1186/1471-2164$\underline{14-857}$

Chinese Pharmacopoeia Commission (2015). Pharmacopoeia of the People's Republic of China Vol. I. China Medical Science Press, Beijing, 26.

Chung HS, Um JY, Kim MS, Hong SH, et al. (2002). Determination of the site of origin of Pinellia ternata roots based on RAPD analysis and PCR-RFLP. Hereditas 136: 126-129. http://dx.doi.org/10.1034/j.1601-5223.2002.1360206.x

Conesa A, Götz S, García-Gómez JM, Terol J, et al. (2005). Blast2GO: a universal tool for annotation, visualization and analysis in functional genomics research. Bioinformatics 21: 3674-3676. http://dx.doi.org/10.1093/bioinformatics/ $\underline{\text { bti610 }}$

Gao JP, Wang D, Cao LY and Sun HF (2015). Transcriptome sequencing of Codonopsis pilosula and identification of candidate genes involved in polysaccharide biosynthesis. PLoS One 10: e117342. http://dx.doi.org/10.1371/journal. pone. 0117342

Grabherr MG, Haas BJ, Yassour M, Levin JZ, et al. (2011). Full-length transcriptome assembly from RNA-Seq data without a reference genome. Nat. Biotechnol. 29: 644-652. http://dx.doi.org/10.1038/nbt.1883

Han L, Chen C, Wang B and Wang ZZ (2015). The complete chloroplast genome sequence of medicinal plant Pinellia ternata. Mitochondrial DNA 1-2 10.3109/19401736.2015.1060441.

Han M, Yang X, Zhang M and Zhong GY (2006). Phytochemical study of the rhizome of Pinellia ternata and quantification of phenylpropanoids in commercial Pinellia tuber by RP-LC. Chromatographia 64: 647-653. http:// dx.doi.org/10.1365/s10337-006-0103-8

Hao D, Ma P, Mu J, Chen S, et al. (2012). De novo characterization of the root transcriptome of a traditional Chinese medicinal plant Polygonum cuspidatum. Sci. China Life Sci. 55: 452-466. http://dx.doi.org/10.1007/s11427-012$\underline{4319-6}$

Huadong W and Faming W (2012). Investigation of Pinellia ternata resources in China. J. Anhui Agr. Sci. 40: 150-151.

Huse SM, Huber JA, Morrison HG, Sogin ML, et al. (2007). Accuracy and quality of massively parallel DNA pyrosequencing. Genome Biol. 8: R143. http://dx.doi.org/10.1186/gb-2007-8-7-r143

Iseli C, Jongeneel CV and Bucher P (1999). ESTScan: a program for detecting, evaluating, and reconstructing potential coding regions in EST sequences. ISMB 99: 138-148. 
Kewei Z, Hao W and Luling W (2002). Fatty acid composition of research in Pinellia medicinal materials. J. Nanjing TCM Univ 18: 291-292.

Li D, Deng Z, Qin B, Liu X, et al. (2012). De novo assembly and characterization of bark transcriptome using Illumina sequencing and development of EST-SSR markers in rubber tree (Hevea brasiliensis Muell. Arg.). BMC Genomics 13: 192. http://dx.doi.org/10.1186/1471-2164-13-192

Li T, Li M, Jia J, Wu FM, et al. (2009). Survey of national resources and production status of Pinellia ternata in China. Res. Pract. Chin. Med. 2: 007.

Li Y, Liu X and Zhu Z (2004). Progress on the research of pharmacology of Pinellia ternata. J. Liaoning Coll. Trad. Chin. Med. 6.

Lin J, Zhou X, Gao S, Wu W, et al. (2006). Authentication of Pinellia ternata and its adulterants based on PCR with specific primers. Planta Med. 72: 844-847. http://dx.doi.org/10.1055/s-2006-931605

Liu LL, Yang ZJ, Wei SH, Ouyang ZM, et al. (2012). ISSR and SRAP markers in the genetic relationship analysis among Pinellia in China. J. Med. Plants Res. 6: 3596-3602.

Liu Y, Liang Z and Liu J (2010a). Use of protocorm-like bodies for studying alkaloid metabolism in Pinellia ternata. Plant Cell Tissue Organ Cult. 100: 83-89. http://dx.doi.org/10.1007/s11240-009-9622-y

Liu Y, Liang Z and Zhang Y (2010b). Induction and in vitro alkaloid yield of calluses and protocorm-like bodies (PLBs) from Pinellia ternata. In Vitro Cell. Dev. Biol. 46: 239-245. http://dx.doi.org/10.1007/s11627-009-9268-9

Lu H, Xue T, Zhang A, Sheng W, et al. (2013). Construction of an SSH library of Pinellia ternata under heat stress, and expression analysis of four transcripts. Plant Mol. Biol. Rep. 31: 185-194. http://dx.doi.org/10.1007/s11105-012$\underline{0488-5}$

Lulin H, Xiao Y, Pei S, Wen T, et al. (2012). The first Illumina-based de novo transcriptome sequencing and analysis of safflower flowers. PLoS One 7: e38653. http://dx.doi.org/10.1371/journal.pone.0038653

Luo C, Wu HX, Yao QS, Wang SB, et al. (2015). Development of EST-SSR and TRAP markers from transcriptome sequencing data of the mango. Genet. Mol. Res. 14: 7914-7919. http://dx.doi.org/10.4238/2015.July.14.17

Luo Q, Xiao Y, Wang K and Yang L (2013). Analysis on the genetic control of heterosis for guanosine and organic acid contents in tuber of Pinellia ternata by using of random amplified polymorphic DNA (RAPD) makers. J. Med. Plants Res. 7: 857-862.

Margulies M, Egholm M, Altman WE, Attiya S, et al. (2005). Genome sequencing in microfabricated high-density picolitre reactors. Nature 437: 376-380.

Natale DA, Shankavaram UT, Galperin MY, Wolf Yi, et al. (2000). Towards understanding the first genome sequence of a crenarchaeon by genome annotation using clusters of orthologous groups of proteins (COGs). Genome Biol. 1: RESEARCH0009.

Novaes E, Drost DR, Farmerie WG, Pappas GJ, Jr., et al. (2008). High-throughput gene and SNP discovery in Eucalyptus grandis, an uncharacterized genome. BMC Genomics 9: 312. http://dx.doi.org/10.1186/1471-2164-9-312

Tatusov RL, Galperin MY, Natale DA and Koonin EV (2000). The COG database: a tool for genome-scale analysis of protein functions and evolution. Nucleic Acids Res. 28: 33-36. http://dx.doi.org/10.1093/nar/28.1.33

Temnykh S, DeClerck G, Lukashova A, Lipovich L, et al. (2001). Computational and experimental analysis of microsatellites in rice (Oryza sativa L.): frequency, length variation, transposon associations, and genetic marker potential. Genome Res. 11: 1441-1452. http://dx.doi.org/10.1101/gr.184001

Varshney RK, Graner A and Sorrells ME (2005a). Genic microsatellite markers in plants: features and applications. Trends Biotechnol. 23: 48-55. http://dx.doi.org/10.1016/j.tibtech.2004.11.005

Varshney RK, Sigmund R, Börner A, Korzun V, et al. (2005b). Interspecific transferability and comparative mapping of barley EST-SSR markers in wheat, rye and rice. Plant Sci. 168: 195-202. http://dx.doi.org/10.1016/j. plantsci.2004.08.001

Wang Z, Fang B, Chen J, Zhang X, et al. (2010). De novo assembly and characterization of root transcriptome using Illumina paired-end sequencing and development of cSSR markers in sweet potato (Ipomoea batatas). BMC Genomics 11: 726. http://dx.doi.org/10.1186/1471-2164-11-726

Xu T, Wang B, Liu X, Feng R, et al. (2012). Microarray-based identification of conserved microRNAs from Pinellia ternata. Gene 493: 267-272. http://dx.doi.org/10.1016/j.gene.2011.08.009

Yang L, Ding G, Lin H, Cheng H, et al. (2013). Transcriptome analysis of medicinal plant Salvia miltiorrhiza and identification of genes related to tanshinone biosynthesis. PLoS One 8: e80464. http://dx.doi.org/10.1371/journal. pone. 0080464

Yao J, Pang Y, Qi H, Wan B, et al. (2003a). Transgenic tobacco expressing Pinellia ternata agglutinin confers enhanced resistance to aphids. Transgenic Res. 12: 715-722. http://dx.doi.org/10.1023/B:TRAG.0000005146.05655.7d

Yao JH, Zhao XY, Liao ZH, Lin J, et al. (2003b). Cloning and molecular characterization of a novel lectin gene from 
Pinellia ternata. Cell Res. 13: 301-308. http://dx.doi.org/10.1038/sj.cr.7290175

Ye J, Fang L, Zheng H, Zhang Y, et al. (2006). WEGO: a web tool for plotting GO annotations. Nucleic Acids Res. 34: W293-7. http://dx.doi.org/10.1093/nar/gk1031

Yu JK, La Rota M, Kantety RV and Sorrells ME (2004). EST derived SSR markers for comparative mapping in wheat and rice. Mol. Genet. Genomics 271: 742-751. http://dx.doi.org/10.1007/s00438-004-1027-3

Yueqin H (2008). Study on the development and differentially expressed genes of small tuber of Pinellia ternata in tube. Master's thesis, Hua Zhong Agricultural University, China.

Zhang XF, Li J, Qi G, Wen K, et al. (2011). Insecticidal effect of recombinant endophytic bacterium containing Pinellia ternata agglutinin against white backed planthopper, Sogatella furcifera. Crop Prot. 30: 1478-1484. http://dx.doi. org/10.1016/j.cropro.2011.07.012 